\title{
Use of retrograde urethrography in the resuscitation room
}

Sir,

It is now generally accepted that the management of patients in the Accident Department suffering with multiple injuries should follow the guidelines of the Advanced Trauma Life Support course (American College of Surgeons, 1989). The guidelines for the management of patients with suspected urethral or bladder injuries advise that an ascending urethogram be performed to assess the integrity of the tract.

We have found this technique to be easy to perform on the trauma patient and to yield useful diagnostic information with minimal expenditure of time. We use a 12 Foley catheter anchored in the fossa navicularis by gentle insufflation of the balloon using $2-3 \mathrm{ml}$ of water. It is important to converse with the conscious patient when this is being performed, the technique is well tolerated if adequate explanation is given. Between 50 and $100 \mathrm{ml}$ of contrast material is injected through the main channel of the catheter using a bladder syringe. The quantity of dye needed will vary according to the build of the patient and the film should be exposed during the active injection of contrast material to produce a dynamic retrograde urethrogram. Care must be taken not to spill any contrast material and to maintain a good connection between the syringe and catheter otherwise interpretation of the films may be difficult. Although in general the results of the urethrogram are fairly easy to interpret, liaison with the radiologist is advocated.

An oblique urethrogram is preferred because in the antreroposterior projection the bulbous urethra is foreshortened and can cause difficulty in interpretation. With a patient on a casualty trolley who has multiple injuries with possible splintage of limb injuries it may be impossible to tilt the patient. The same effect can be achieved by offsetting the film underneath the patient and tilting the beam source (Fig. 1). Having obtained suitable films and if there is no contraindication that the balloon can be deflated and the catheter gently passed into the bladder.

It is a particularly useful investigation in patients who may have blunt abdominal trauma or pelvic fractures who will need to be catheterized anyway prior to performing a peritoneal lavage. A rectal examination is of course mandatory prior to attempted catheterization to assess the position of the prostate, rectal tone and to detect the presence of any blood in the rectum which may suggest a rectal tear.

In conclusion we have found ascending urethrography an easy procedure to perform in the resuscitation room yielding valuable information for minimal expenditure of time.

\author{
M. J. WILLIAMS \& M. J. CLANCY \\ Accident \& Emergency Department \\ Hull Royal Infirmary \\ Hull, U.K.
}




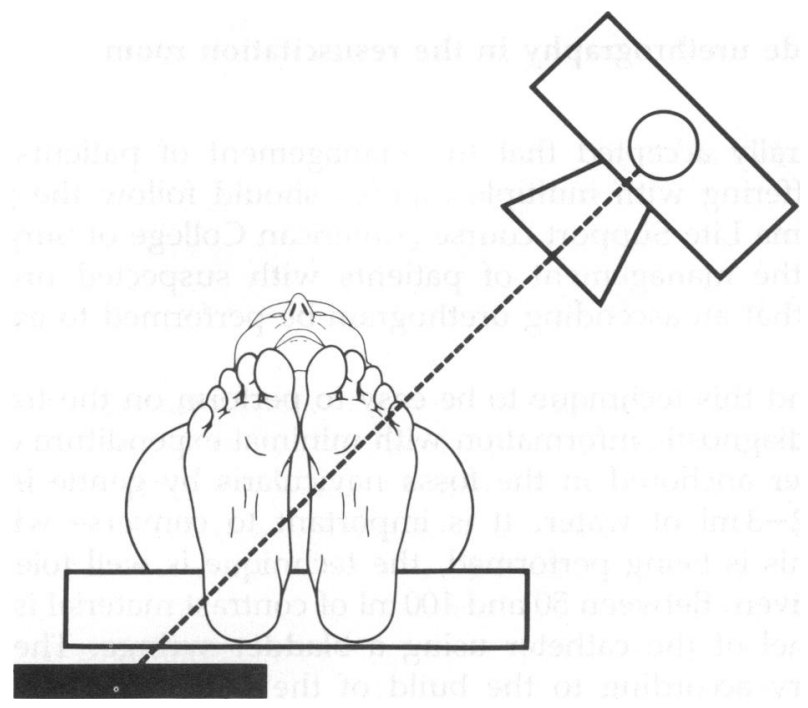

Fig. 1. Offsetting the film plate and tilting the $X$-ray beam to obtain an oblique view without having $\vec{C}$ to move the patient.

\section{REFERENCE}

Advanced Trauma Life Support Student Manual (1989). American College of Surgeons, Chicago, Illino高. 